\title{
Automated truck design for glass-waste collection with optimised compactor for glass recycling
}

\author{
M. Zordan \& S. Morassut \\ Regola Team Srl, Porcia (PN), Italy
}

\begin{abstract}
Waste recycling begins from the collection phase. The ordinary activity of emptying bins in trucks for subsequent stocking, material separation and recycling treatment has great impact over the whole process efficiency. With optimised garbage collection it is possible not only to achieve a better recycling rate in terms of quantity and material quality, but also to deeply cut the costs of material selection and treatment in the recycling process. This especially applies to collected glass where the recycling steps require multiple treatments for sorting, processing and glass re-use either with "new" material, to obtain glassbased items, or mixed to other substances, even to produce completely different composites. This paper highlights some "design guidelines" of a truck aimed at urban glass-waste collection. Two features give unique capabilities to the vehicle: the automation of both loading and compacting processes and the compactor performance with regard to the size of the treated material, cut down in pieces suitable for optimal recycling efficiency.

Keywords: compactor, glass, recycling, truck, vehicle, fieldbus.
\end{abstract}

\section{Introduction}

\subsection{Waste collection}

Garbage collection involves many interactions among technical and organisational aspects. Logistic problems concentrate in the management of a fleet of vehicles (trucks, loaders, etc.) and in the coordination of the related personnel. The decision where to set collection points (waste bins, containers, etc.) is a trade-off between user commodity, time spent for collection and 
foreseen waste quantity. Typical parameters to be evaluated in such decisions (such as habitants/area, bins/road-length, habitants/blocks) must be weighted by correction factors taking into account the distribution of residential and industrial zones in the same neighbourhood. Even if urban garbage collection is mainly related to domestic waste, special collections must also be considered for demolition waste and heavy/cumbersome materials especially concentrated around commercial premises.

Technical matters relate to waste separation, stocking and disposal (dump, landfill). Material treatment is then an issue when recycling processes are to be carried on from collected waste. Cities usually differentiate various materials: aluminium, glass, plastic, paper, organic material. Many of them undergo recycling treatment while the remaining is disposed in landfill sites.

\subsection{Glass-waste collection}

Glass-waste, together with paper-waste is the first material that has been considered for recycling and the related technology is nowadays mature. Glasswaste collection is usually carried out using different types of containers: "bell bins" (containers with openings on their sides to insert the disposed glass, which are emptied through their bottom, once they are lifted over a collecting truck) and "wheelie bins" (relatively small plastic containers with limited capacity, 120-240 litres). "Bell bins" usually serve wide areas (blocks, streets, etc., corresponding to about $600 \mathrm{~m}$ in size) while "wheelie bins" are suited for domestic needs (they could be placed every ten/twenty residential units, or about 50-100 $\mathrm{m}$ from each other). It is easier to collect from wheelie bins because:

- reduced waste quantity requires small vehicles to be used for collection;

- small bin dimension facilitates the loading phase (which is comparable to the collection of "general" waste);

- safety issues are reduced due to small quantity/weight of material being raised and emptied on collecting vehicles;

- the loading phase is much more suitable to be performed automatically;

- less time required per loading allows a smoother collection phase to be performed in busy road traffic conditions.

Therefore the trend in glass-waste collection is towards allocation of many collection spots ("wheelie bins"), especially in the city centre, instead of concentrating the collection in "bell bins" (usually kept in the suburbs).

The truck whose design guidelines are presented in this work is intended for glass-waste collection using "wheelie bins". The vehicle must perform the collection together with the compacting action aimed at reducing the glass in fragments suitable to be recycled afterwards (about $5 \mathrm{~cm}$ in size). If glass fragments exceed that dimension, an additional step in the recycling process must be performed, aimed at crumbling the material to make it suitable for the following treatments. On the other hand if glass is broken down into too small pieces, it becomes difficult to separate from other materials and to manage in the waste disposal area. The compacting action avoids to perform the crumbling process once the glass is stocked and it also makes easier the separation of 
different materials (plastic bags, aluminium tops, etc.) sometimes collected with the glass: the compactor doesn't cut in small fragments non-glass material.

\section{Compactor mechanical design}

\subsection{The container}

The truck hosts a container to store the collected glass and to hold the material during the compacting process: this is necessary in order to fragment the glass in parts whose dimensions are suited for later treatment.

The container is made of a single iron sheet metal, and it has the inside covered with anti resound paint, in order to prevent both loud acoustic noise during loading operations and exposure of the metal structure to corrosive climatic agents. The supporting structure is an iron frame at the compactor edges: no other strengthening elements have been considered, in order to obtain a light but strong metal structure. The container section is rounded towards the outside of the structure: this evenly distributes the containing forces and helps the fragmented glass to compensate the pressing forces by "going around" the compactor mechanical parts (details in section 2.3). The truck weight is actually a key-element of the design as the lighter is the structure, the larger becomes the container capability.

Various unloading alternatives have been studied. The most effective one (considered in the truck) requires the back of the container to open while the container itself rises on the front part: the collected glass therefore drops down by gravity and accumulates behind the truck (which can drive straight on once the unloading has been completed). This is achieved with the back of the container hinged at its upper side while the container rises thanks to an oleodynamic cylinder lifting its front (figure 1). As the back of the container acts also as contrasting structure to the compactor pressure (details in section 2.2), great care has been spent on the fastening blocks. A two-movement lock has been designed: with a single movement, the back of the container at first rises up unlocking the container and then rotates to open position (figure 2).

\section{2 "Slider" and "Shovel"}

The compacting force presses the collected glass against the rear part of the container. This is achieved using two moving mechanical parts: a "slider" and a "shovel". Their joint movements also ensure the glass remains confined for enhanced vehicle stability.

1) The slider is a horizontal support that moves from the front to the back of the container. It holds the shovel and it is driven by oleodinamic cylinders.

2) The shovel (figure 3) rotates around hinges fixed to the slider and it presses the collected glass. It is driven by oleodinamic cylinders.

To obtain even grinding of the collected glass, the mechanical parts have been designed appropriately: an optimised choice of their joint movements, with regulated pressure against the material, yields the desired result. 

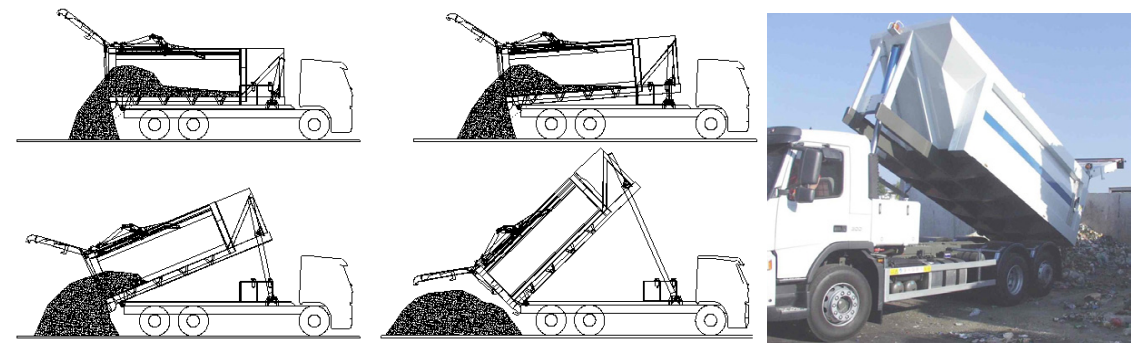

Figure 1: $\quad$ Truck unloading.
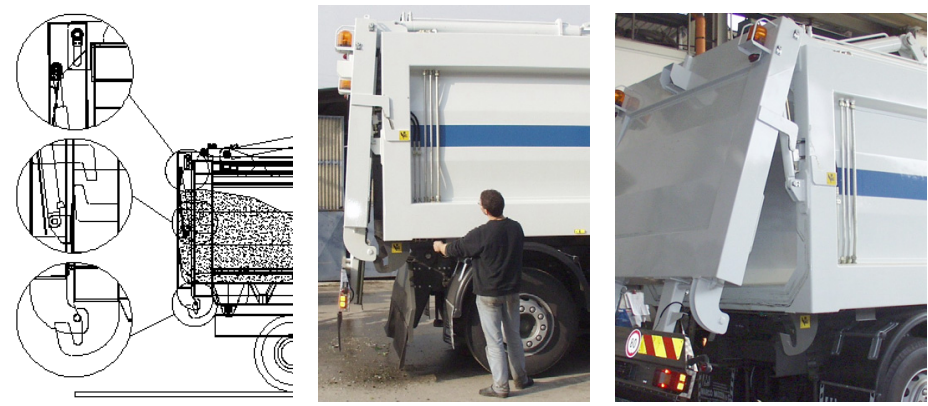

Figure 2: Container locking elements.

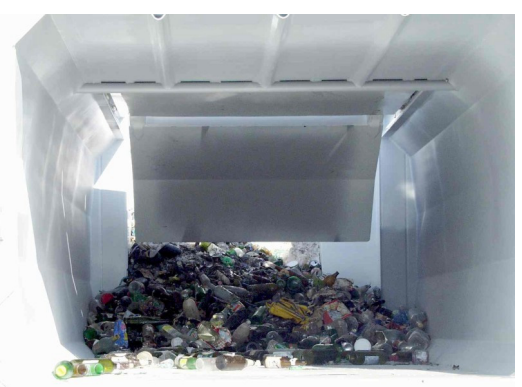

Figure 3: Shovel seen from the back of the container.

\subsection{Compactor movements}

Extensive simulation analysis has been carried on, considering the movements of the slider and the shovel as separate actions. The fist one (slider moving with the shovel in vertical position) presses the glass against the back of the container while the second one (shovel rotation) presses the glass against the bottom of the container. To ensure uniform crumbling of the whole collected glass, a sliding movement, with uniform pressure of the shovel against the glass, has to be performed (figure 4). The slider moves from the front of the container holding 
the shovel in vertical position. This collects the glass which has just been loaded (if any) to the rear part of the container. As soon as the shovel starts compressing the glass with a force exceeding a chosen value (sensed on the oleodynamic pipe), its driving cylinders are released making the shovel stop pressing the glass. As the slider keeps moving, the shovel will soon compress the glass with greater pressure than allowed, turning on again the regulating effect. This results in a continuous sliding movement with uniform pressure against the glass. To smooth the pressure of the compactor and to make it more uniform on the whole collected glass, the shovel has been designed with reduced dimensions with respect to the inside of the container (figure 3). This makes the glass move through escaping paths around the lateral edges of the shovel if uneven pressure is applied. The regulation effect of this solution results in very effective, uniform glass crumbling, better than acting only with precise regulation of the oleodynamic forces on both the slider and the shovel. The tuning of the oilpressure power circuitry is still necessary for a fine glass treatment. Practical tests on a prototype confirmed the quality of the compacting action and the uniform fragmentation of the collected glass. Force adjustments can also be made by setting the pressure value at which the shovel force is released.
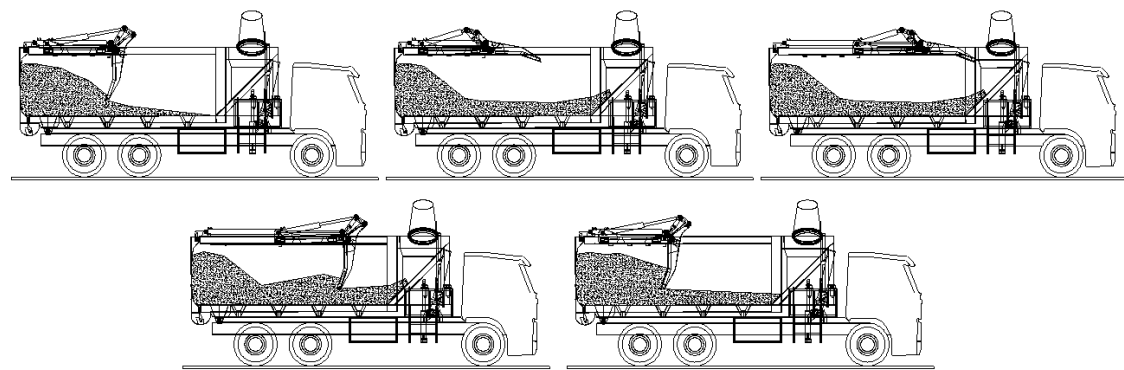

Figure 4: Compactor movements.
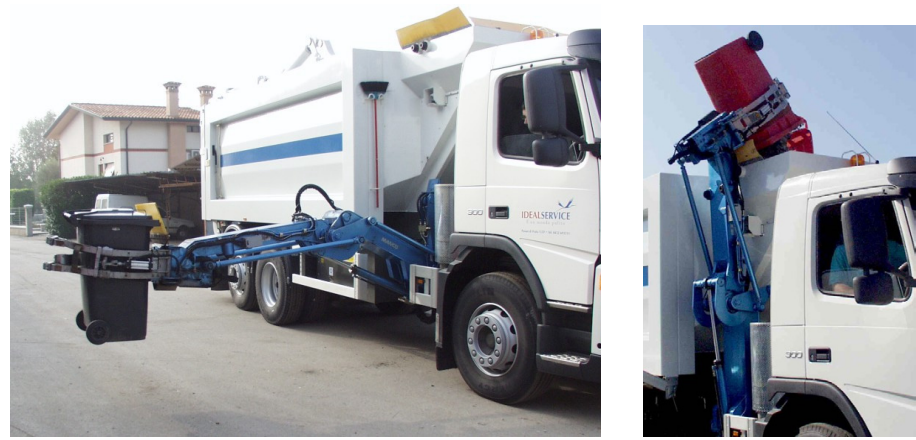

Figure 5: Loading operation. 


\subsection{The loading arm}

A side loader has been considered to empty the wheelie bins in the container. Its position allows fast and prompt collection of the bins on the pavements. The loading arm is bent in the middle so that it remains in folded position when it is retreated on the vehicle. The outer end is provided with two holders that take the bin at its sides (after the arm is extended with horizontal movement); the arm then lifts it up over the container making the glass fall down on the inside (figure 5). This movement also opens the bin cover and set it to closed position once the bin is put back in its original place. For intervention on standard pavements the loading arm is required to extend to about $3 \mathrm{~m}$. To minimise the space occupied by the loading arm in closed potion, one of its grabbing ends is folded, and it is automatically extended during operation.

\section{Vehicle operating specifications}

The main characteristics of the glass collection vehicle are listed hereafter and they result from a trade-off between easiness of use, compliance of actual regulations (garbage treatment), safety at work and effectiveness of glass pretreatment (installed compactor).

1) The vehicle has to be operated by a single person, even if two operators are expected to speed up the collection.

2) The loading phase must be independent from the compacting phase.

3) The operator must control every appliance from the driving cabin.

4) Dangerous operations (directly on the compactor and/or on the loading arm, outside the driving cabin) must be executed with "two hands" commands.

\section{Automation system}

The truck has been provided with sensors and actuators for automatic operation of all mechanical systems. The centralised control unit, in the cabin, allows the operator to control the bin loading arm and the glass compactor with simple commands on a comfortable user interface (graphic display with touch screen). Once the bin is aligned with the loader, the operator has to simply press a button and the glass is collected without any further human intervention.

Such a control system has been designed using traditional computational devices (industrial PC), running executable code programmed using high level language in order to exploit at best the computational resources. It can be easily adapted to other trucks operating garbage collection and, in general, to vehicles fitted with tanks and containers to carry and treat specific materials (liquid sewage, mud and water discharge transport, etc.). In similar situations the devices dedicated to specific tasks are mounted onto the truck structure; if necessary, the vehicle provides the mechanical power (through the drive shaft connected to the engine) and the electrical power supply (batteries and energy generation system). 


\subsection{The fieldbus}

The system uses a fieldbus interconnection to provide an efficient interface to sensors and actuators. Moreover, the fieldbus allows optimal cabling in terms of used space and intervention time required for both installation and maintenance. With regard to the devices mounted on the truck, they are:

- sensors (such as mechanical transducers, for both angular and linear movements, proximity, level, temperature, etc.)

- actuators (such as oleodynamic and pneumatic electric valves, mechanical releasers, relais commands, signalling lamps, etc.)

Employed sensors are:

- inductive proximity transducers have been positioned at the ends of the moving zones of every mechanical component;

- $\quad$ encoders sense the absolute position of the loading arm in its two joints;

- $\quad$ ultrasonic meters provide the distance of the bin from the truck.

Every sensor has its signal output: proximity transducers give on/off status with binary digital signal output, ultrasonic distance meters treat continuous information and thus they interface through analogue output voltage, complex devices require the information to be supplied using multiple signals and/or to be digitized and transferred through particular protocols.

Actuators act on the devices by delivering electric and pneumatic commands. In the truck the whole oleodynamic system is governed by electric valves and therefore digital (on/off) and analogue (continuous over the whole output range) power outputs are required; the grabbing ends of the loading arm are extended through pneumatic commands.

Among various fieldbus analysed, the Actuator/Sensor Interface (AS-I) has been considered due to its simple device management, flexible supported network structure, easy cable interconnection and certified safety capability integrated in the transmission protocol. The datarate supported by this fieldbus standard, well fits the requirements for interconnection to the devices mounted on the truck, where mainly small packets of information have to be delivered between the sensors / actuators and the central processing unit (industrial PC interfaced through AS-I master). Moreover this multi-vendor open standard makes available on the market a large amount of compliant devices, from generic interfaces to specific components (figure 6). Among them, absolute encoders with direct AS-I interface have been used and easily snapped to the fieldbus cable without any additional interface.

The device power supply is also facilitated by the two-wires "data and power" yellow cable which holds up to 8 amps. If more power is required by consuming devices (such as some electric valves employed in the truck) an additional black cable delivers the requested energy without any further burden affecting data transmission. The advantage of the AS-I solution in case of failures is enhanced by the integrated fault reporting feature implemented in the protocol: corrective actions have been programmed to promptly face the faults by driving the system to a safe shutdown or to safe positions. Once the faulty device is located, its simple replacement restores the full system efficiency being 
the fieldbus protocol in charge of re-configuring the new device with the same parameters of the previous one. This feature has been greatly appreciated by maintenance teams which can operate with shorter intervention times (that have been cut down to an average $60 \%$ with respect to conventional systems).

The AS-I standard includes the implementation of a safety protocol ("Safety at Work") which has been approved for applications up to Category 4 according to EN 954-1 and it also meets the requirements of Safety Integrity Level 3 (SIL3) of IEC 61508 on safe transmission.
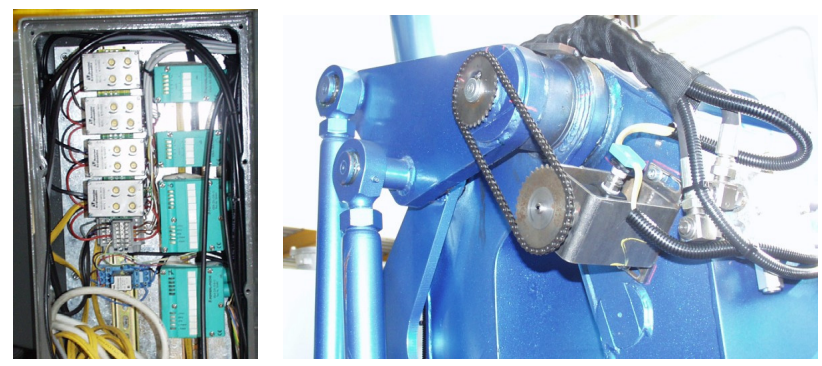

Figure 6: AS-I sensor / actuator interface devices and encoder

\subsection{Monitoring and controlling software}

The monitoring and controlling procedures (parameterised for full configurability) are structured in modules (libraries) and performed by software executed on standard operating systems. This helps the operator intervention, being also facilitated by the provided touch screen (tested for glove wearing users, as happens during waste collection) for direct interaction with the displayed interface elements, buttons and controls (figure 7).

Accurate software programming allows the implementation of concurrent control processes (multithread programming) helping the execution of selfcontained routines, independent from one another: for example it is possible to verify the device status and to drive actuators meanwhile the control algorithm manages the system. Three main modules have been considered: implementation of the operating procedures for control and system configuration, fieldbus management, user interface management.

\subsubsection{Module implementing the operating control procedures and the system configuration}

It detects sensors and actuators, it recognises and matches their capabilities to the operating functionalities of the glass-waste collection truck. It resolves every addressing issue related to the communication through the fieldbus and it identifies every device task (for example it relates a movement limit to its corresponding sensor, an electric valve to an actuator, etc.). If faults are detected, not allowing safe execution of every system functionality (such as the lack of detection in the reach of a mechanical limit), the software enters a "user warning mode" thus allowing problem identification and solving. 
The module also implements the following procedures for the control of the loading arm and the glass compactor.

- Automatic loading: the operator aligns the truck to the bin and he starts the collection. Automatically the arm extends to reach the bin, it grabs it and empties it into the container. Afterwards the arm puts down the bin in its original place and it returns on its rest position.

- Manual loading: the operator switches the system to use a joystick to control the loading arm (figure 7). This is useful if waste bins are in wrong positions, inaccessible by the standard automatic procedure, but at the same time it avoids the operators to go out of the driving cabin and manually move the bin in a correct position.

- Semi-automatic loading: the operator can grab manually the bin and the automatic procedure completes the loading operation.

- Compaction: the operator can start a compacting action. It is even possible to set the compactor to act automatically after every collection.

- Unloading: the operator can manage the container unloading procedure according to applicable safety regulations.
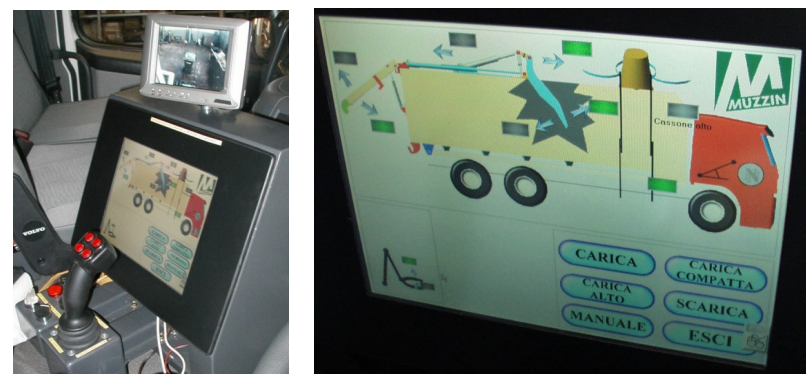

Figure 7: $\quad$ Operator interface (touch-screen display and joystick).

\subsubsection{Module implementing the fieldbus management}

Data acquisition and command delivering is performed through the AS-I master device connected to the fieldbus: the interconnection routines have been optimised for speed without compromising error and data consistency checks. Remedial and/or recovering actions can be triggered in case of fault detection.

The possibility to access the fieldbus (retrieving data and/or sending commands) is granted independently from the corresponding function calls in the control software. For example this allows more than one control process to access the fieldbus through the same AS-I master device, provided that the different processes act on different sets of slave devices, otherwise contention errors may occur. This capability has been used in the independent control of the loading arm and the compactor.

\subsubsection{Module implementing the user interface}

It manages the user interaction and commands execution through the industrial PC display. Showing the system status can be effective if data are organised in 
graphic form, thus allowing a fast and easy way to recognise the configuration of the installed devices and the instantaneous position of every mechanical part of the loading arm and the glass compactor (figure 7). This software module also performs the initial setup of the control program, the insertion of the operating parameters and the checking of the executed actions (e.g. using graphical views, $\log$ files, etc.). If anomalous running conditions are detected, or unaccepted system configurations are set, it also activates warning alarms.

The complete monitoring of the controlling software (also saving data in a "log file") is essential in order to keep track of the use of every vehicle (waste collectors, compactors, etc.) which is part of the fleet dedicated to garbage collection. Thanks to the module structure of the software, additional improving functions could be easily implemented: for example remote data collection can be considered by using medium-long wireless interconnection, with values stored in a comprehensive database for later evaluation. Remote monitoring could address vehicle routing and locating issues (logistic), loading optimisation by evaluating the weight of collected glass in real time, etc.

The automated system allows a single operator to drive the truck and, at the same time, to manage the waste collection. In practice, also in order to support other activities required during the collection, two operators are employed. Nevertheless it has been confirmed that the operators' burden during glass-waste collection is greatly reduced together with the risks involved by this task.

\section{Conclusions}

This paper presented some design guidelines related to a glass-waste collection truck with integrated compactor. The collection is achieved using a side-loading arm controlled by an industrial-PC based system that includes a fieldbus for fast command delivering and system monitoring. A suitable, customised software manages the whole system, in which the two parts, compactor and loading arm, are actuated either synchronously or independently from each other.

Few operators are required: the complete collection route can be entirely completed by one person only, the driver of the vehicle. In practice two operators are sufficient and manual intervention is reduced to a minimum.

The optimisation achieved in the compactor design gives optimal glass fragmentation for recycling: this results from the mechanical design of the compactor shovel and the tuning of its oil-pressure power circuitry.

\section{Acknowledgments}

The authors gratefully acknowledge Muzzin Srl for the precious technical advices during the design and prototyping stages of the work, and Pepperl+Fuchs Italy for the AS-I fieldbus support. 